\title{
XXVII. Meerschaum von Eskishehir in Kleinasien.
}

\author{
Von
}

E. Weinschenk in München.

Die Zusammensetzung des Meerschaums ist insofern noch nicht vollständig klar gestellt, als die Analysen dieses Minerals in Beziehung auf den Wassergehalt sehr verschiedene Resultate ergaben, ohne dass mit Sicherheit die Ursache dieser Unregelmässigkeit nachgewiesen worden wäre. Eine erneute Untersuchung wurde daher von Herrn Prof. G rot b angeregt, als einige Prachtstufen von Meerschaum durch die freundliche Vermittelung von Herrn E. Naumann aus den altbekannten Gruben bei Eskishehir zwischen Brussa und Angora an der anatolischen Bahn in Kleinasien an die kgl. bayerische Staatssammlung gelangten.

Ueber das Vorkommen des Meerschaums in Kleinasien wurde schon mannigfach berichtet, und Herr E. Naumann ${ }^{1}$ ) bestätigte vollkommen die Angaben uber das Auftreten desselben in zackigen und eckigen Putzen in einem zähen, braunrothen Letten, welcher durch Austrocknen hart und rissig wird. Derartige Ablagerungen treten ausser bei Eskishehir noch an verschiedenen Stellen in Kleinasien auf; sie stehen nach E. Naumann allenthalben in Beziehung zu Serpentinhugeln, welche durch das Vorkommen ungemein zahlreicher Magnesitgänge und Chromitputzen ausgezeichnet sind, aber nur selten gelingt es, den Meerschaum auf primärer Lagerstătte im Serpentin zu beobachten, mit dessen Entstehung er sicher in Zusammenhang gebracht werden muss. E. Naumann beobachtete Derartiges östlich von Erenkiōi am Ausgebenden einiger Magnesitgänge, doch werden auch dort ausschliesslich die Vorkommnisse auf secundärer Lagerstätte aus gebeutet.

Ueber die Entstehung des Meerschaums lassen sich mit Ausnahme der sicher nachweisbaren genetischen Beziehungen zum Serpentin keine Anhaltspunkte gewinnen, doch scheint es an sich unwahrscheinlich, dass der-

1) E. Na umann, Vom Goldenen Horn zu den Quellen des Euphrat. München und Leipzig 1893. X. Capitel. Die Meerschaumg!uben. 
selbe durch Umbildung aus dem Magnesit hervorgegangen sein soll, indem einestheils die äusserst dichte und compacte Beschaffenheit des Magnesits circulirenden Lösungen nur schwierig den Durchgang gestatten würde, anderntheils die feinporige Textur des Meerschaums selbst so ausserordentlich charakteristisch und gleichbleibend ist, was wohl kaum auf ein besonders compactes Muttermineral schliessen lässt. Vielmehr ist anzunehmen, dass der Meerschaum eine dem Magnesit durchaus gleichwerthige Bildung darstellt, welche ebenso wie dieser mit postvulkanischen Processen in Verbindung gebracht werden muss.

Auch die mikroskopische Untersuchung liefert keine neuen Gesichtspunkte. Unter dem Mikroskope erscheint das Mineral als ein sehr dichtes Aggregat von fasriger, oft etwas sphärolithenartiger Beschaffenheit, der Grundmasse gewisser Quarzporphyre nicht unähnlich, dessen einzelne Elemente schwache Doppelbrechung aufweisen; doch lassen sich bei der Kleinheit derselben weitere optische Bestimmungen nicht durchführen. Auch die Form der Poren entzieht sich der mikroskopischen Beobachtung vollsıändig, was vielleicht mit der geringen Grösse der letzteren zusammenhängt, vielleicht aber auch mit der Eigenschaft des Meerschaums, beim Schleifen oder Schneiden zu schmieren, wodurch überhaupt die Structur desselben undeutlich wird. Aucb durch Imprägnation mit Farbstoffen konnten in dieser Beziebung keine Resultate erlangt werden. Der Meerschaum nimmt, mit alkoholischer Lösung von Farbstoffen im Vacuum behandelt, dieselben zum Theil (Eosin, Pikrinsäure, Brillantgrün) mit grosser Leichtigkeit und sehr gleichmässig auf, zum Theil zieht er aus den Lösungen (Methylenblau) den Alkohol aus und lagert den Farbstoff in scharf abgegrenzten Randzonen in concentrirter Form ab, welche sich nur bei längerem Behandeln im Vacuum verbreitern, stets aber durch eine scharfe Linie von dem ungefärbten, aber mit Alkohol vollgesogenen Kerne sich abgrenzen.

Von Salzsäure wird der Meerschaum unter Ausscheidung gallertiger $\mathrm{SiO}_{2}$ leicht zersetzt, ebenso lösen ihn Alkalien schon bei gewöhnlicher Temperatur auf. Mit Wasser quillt or bedeutend auf und lässt sich dann zu einer seifenartigen, plastischen Masse zerreiben.

Im Kölbchen giebt das lufttrockene Material schon unter 1000 viel Wasser ab, bleibt aber in seinen optischen Verbältnissen auch dann vollständig unverändert, wenn es lang andauernd bei $300^{\circ}$ getrocknet worden ist. Bei dunkler Rothgluth geht wieder eine bedeutende Menge Wasser weg, und nun ist der Rückstand vollständig amorph geworden; endlich schmilat das Mineral äusserst schwierig an den Kanten zu weissem Email.

Die Erscheinung, dass durch den sehr bedeutenden Wasserverlust bei $100^{\circ}$ eine Aenderung der optischen Eigenschaften nicht eintritt, halte ich für einen unzweifelhaften Beweis dafür, dass das bei dieser Temperatur weggehende Wasser mit der Constitution des Minerals nichts zu thun hat, 
sondern vielmehr als bygroskopisch betrachtet werden muss, wie ja schon an sich die äusserst fein poröse Beschaffenheit desselben es wahrscheinlich macht, dass es sehr hygroskopisch ist.

Es wurden einige quantitative Bestimmungen des Gewichtsverlustes bei verschiedenen Temperaturen mit möglichst reinem Material ausgefúhrt. Bei einem ersten Versuche wurden $0,4410 \mathrm{~g}$ vollständig lufttrockner Substanz 48 Stunden lang im Vacuum uber Schwefelsäure behandelt, Gewichtsverlust $0,0633 \mathrm{~g}=14,35 \%$. Im Luftbade bei $110^{\circ}$ verànderte sich das Gewicht nach zweimal sechsstundiger Behandlung nicht weiter; bei $210^{\circ}$ gingen weitere $0,88 \%$ weg, bei $300^{\circ}$ blieb das Gewicht wiederum constant. Ein Theil der Probe wurde nun mikroskopisch untersucht, und es zeigte sicb, wie schon oben bemerkt wurde, dass das Mineral vollständig unverändert war. Zu einem zweiten Versuche wurde Material verwendet, welches mehrere Tage uber Chlorcalcium aufbewahrt worden war; Gewichtsverlust bei $210^{\circ} 9,75 \%$. Ferner wurden $0,3563 \mathrm{~g}$ bei $210^{\circ}$ getrockneter Substanz $\frac{1}{2}$ Stunde lang einem heftigen Gebläsefeuer ausgesetzt, Gewichtsverlust 12,00\%; endlich ergab eine directe Wasserbestimmung von $0,6315 \mathrm{~g}$ bei $210^{\circ}$ getrockneter Substanz durch Erhitzen mit Bleichromat 12, $10 \% \mathrm{H}_{2} \mathrm{O}$. Aus diesen Bestimmungen ergiebt sich unzweifelhaft, dass das unter $300^{\circ}$ weggehende Wasser nur als physikalisch gebunden zu betrachten ist und dem Meerschaum als solchem nicht angehört. Da nun ferner die besseren unter den älteren Analysen übereinstimmend das Verbältniss von $\mathrm{MgO}: \mathrm{SiO}_{2}=2: 3$ angeben, so dass hieruber ein Zweifel uberhaupt nicht besteht, so ergiebt sich als Formel des Meerschaums $\mathrm{H}_{4} \mathrm{Mg}_{2} \mathrm{Si}_{3} \mathrm{O}_{10}$, welche $12,15 \% \mathrm{H}_{2} \mathrm{O}$ verlangt. 